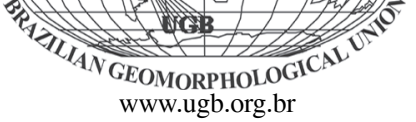

\title{
CLASSIFICAÇÃO DE BACIAS DE DRENAGEM DO ALTO JEQUITAÍ (MINAS GERAIS) A PARTIR DA ANÁLISE DE PRINCIPAIS COMPONENTES E ANÁLISE DE GRUPOS
}

\author{
Robson Veloso Ribeiro Sodré; Osmar Abílio de Carvalho Júnior; \\ Wátila Portela Machado; Sandro Nunes de Oliveira; \\ Roberto Arnaldo Trancoso Gomes; Renato Fontes Guimarães \\ Departamento de Geografia da Universidade de Brasília (UnB) \\ Campus Universitário Darcy Ribeiro, Asa Norte, CEP 70910-900 Brasília, DF \\ \{r_sodré; watilabaixo\}@yahoo.com.br; \\ \{osmarjr;sandronunes; robertogomes;renatofg\}@unb.br
}

\author{
Ana Paula Ferreira de Carvalho \\ Instituto de Colonização e Reforma Agrária- INCRA \\ SBN Quadra 1, Bloco D, Edifício Palácio do Desenvlovimento, sala 1620, CEP 70057-900, Brasília, DF \\ ana.carvalho@incra.gov.br
}

Éder de Souza Martins Embrapa Cerrados - BR 020, km 18, CP 08223, CEP 73310-970, Planaltina, DF eder@cpac.embrapa.br

\begin{abstract}
Resumo
Esse artigo objetiva aplicar a análise multivariada para a descrição e classificação dos dados morfométricos de bacias de drenagem. A metodologia pode ser subdividida em quatro etapas: (a) elaboração do Modelo Digital de Terreno (MDT) hidrologicamente correto, (b) delimitação dos limites da bacia a partir do MDT de acordo com a ordem fluvial de Strahler, (c) determinação dos atributos morfométricos da bacia de drenagem, e (d) análise multivariada (análise fatorial e análise de grupos). A elaboração do MDT considerou os seguintes procedimentos: preenchimento de depressões, aprofundamento da rede de drenagem, direção de fluxo e determinação do fluxo acumulado. As medidas morfométricas das bacias incluíram nove fatores: elevação (média, ponto mais alto e mais baixo), amplitude de elevação (diferença entre o ponto mais alto e baixo na bacia), declividade, curvatura, área, perímetro e índice de circularidade. A análise multivariada foi utilizada para simplificar e organizar a grande quantidade de dados. Como resultados foram encontradas 1.773 bacias de primeira ordem fluvial. 536 de segunda ordem fluvial e 136 de terceira ordem fluvial. A ACP reduziu os atributos morfométricos das bacias de drenagem em três componentes principais, com alta percentagem da variância original. A análise de cluster aglutinou as bacias com padrões morfológicos similares. A classificação das bacias apresenta as unidades geomorfológicas com forte controle geológico.
\end{abstract}

Keywords: Modelo Digital de Terreno; Análise de Grupos; Análise de Componente Principal; Morfometria. 


\begin{abstract}
This paper aims to apply multivariate analysis for drainage-basin morphometry data description and classification. The methodology can be subdivided into four stages: (a) elaboration of the digital elevation model (DEM) hydrologically corrected; (b) catchment boundaries delimitation from DEM according to Strahler stream order; (c) drainage-basin morphometric attributes determination, and (d) multivariate analysis (factor analysis and cluster analysis). The DEM elaboration considered the following procedures: pit filling, stream burning, flow direction and determination of flow accumulation grids. The morphometric measures of basins included nine factors: elevation (mean, highest and lowest point), range elevation (difference between highest and lowest points in the basin), slope, curvature, area, perimeter and circularity index. The multivariate analysis was applied to simplify and organize the large amounts of data. As results were found 1.773 basins of first order streams, 536 of second order streams and 136 of third order streams. The PCA reduced the morphometric attributes of the drainage basins in three principal components, with high percentage of original variance. The cluster analysis agglutinated basins with similar morphological patterns. The basins classification shows geomorphologic units with strong geological control.
\end{abstract}

Keywords: Digital elevation model; Cluster Analysis; Principal Component Analysis; Morphometry.

\section{Introdução}

A geomorfologia gera uma visão integrada do meio físico considerando o conjunto de variáveis responsáveis pela composição da paisagem (Casseti, 1981). Neste contexto, as bacias hidrográficas se configuram como importantes unidades para investigar as características ambientais, uma vez que, os cursos d'água constituem um dos processos mais ativos na formação da paisagem terrestre, estabelecendo um sistema aberto onde há entradas, saídas e transformações (Christofoletti, 1980; Selby, 1985).

As bacias hidrográficas podem ser definidas como áreas de captação natural da água da chuva, com convergência para um único ponto de saída denominado exutório (Coelho Netto, 1995; Silveira, 2001). O seu comportamento hidrológico é moldado em função de suas características geomorfológicas (forma, relevo, área, geologia, rede de drenagem, solo, dentre outros) e do tipo da cobertura natural ou antrópica a qual está submetida (vegetação, uso do solo, etc.) (Lima, 1986).

Desta forma, os modelos de gerenciamento dos recursos hídricos e de estudos ambientais assumem a bacia hidrográfica como a unidade territorial de referência ou de intervenção pelo fato de consistir num dos caminhos preferenciais de boa parte das relações de causa-efeito, principalmente as que envolvem o meio ambiente.

Nos estudos de bacias hidrográficas foram analisados diferentes atributos morfométricos com o objetivo de estabelecer relações e comparações com as dinâmicas do meio ambiente, como: hidrologia, sedimentação, erosão, entre outros. Um dos trabalhos precursores, neste tipo de estudo, foi o de Horton (1945) que adotou a abordagem quantitativa na busca de compreender o desenvolvimento dos cursos d'água e das respectivas bacias. Posteriormente, vários estudos foram realizados dentro dessa concepção (Strahler 1952, 1957; Schumm, 1956). No Brasil, diversos trabalhos adotaram essa abordagem para caracterizar os ambientes e os fenômenos ocorrentes na superfície (Freitas 1952;Tolentino et al., 1968; Christofoletti 1969, 1970, 1977 e 1978; dentre outros).

Com o advento do geoprocessamento os procedimentos para delimitar as bacias hidrográficas e suas métricas apresentam maior rapidez e eficiência. O desenvolvimento dessas métricas e a própria obtenção das bacias está diretamente relacionada com a confecção de um Modelo Digital de Terreno (MDT), que representa matematicamente uma determinada superfície terrestre (Teixeira et al., 1992). A partir de um MDT é possível extrair uma série de atributos do terreno importantes na análise das bacias hidrográficas, tais como: rede de drenagem, desníveis, inclinações, comprimento de canais, orientação de vertentes, direções de fluxo e os limites das bacias e sub-bacias hidrográficas (Doornkamp \& King, 1971; Meijerink, 1988; Moore et al., 1988). Estes diferentes atributos de terreno passíveis de extração automática podem ser classificados por análise multivariada, fornecendo uma compartimentação da paisagem (Giles \& Franklin, 1998; Miliaresis \& Argialas, 1999).

O presente trabalho teve como objetivo identificar as unidades geomorfológicas de uma sub-bacia no alto curso do Rio Jequitaí, a partir da delimitação automatizada de bacias de drenagem e análise multivariada de seus atributos 
morfométricos. Como objetivos específicos têm-se: (a) determinar as bacias conforme as ordens fluviais de Strahler a partir de um MDT hidrologicamente correto; e (b) calcular os índices morfométricos das bacias de drenagem agrupandoos por análise multivariada.

\section{2. Área de Estudo}

A área de estudo encontra-se em Minas Gerais entre as coordenadas UTM 596332 - 637007 Leste e 8016810 - 8083450 Norte, fazendo parte de uma sub-bacia na margem direita do Alto São Francisco, cujo curso principal é o rio Jequitaí (Figura 1).

O clima da região pela classificação de Köppen pertencente à classe $\mathrm{Cw}$, temperado brando com inverno seco. Possui um período chuvoso com influência de massas equatoriais úmidas e um período seco provenientes de massas continentais secas (CODEVASF, 1993).
A área de estudo apresenta como unidades litoestratigráficas o Supergrupo Espinhaço de idade neoproterozóica e o Supergrupo São Francisco de idade permocarbonífera (Figura 2).

O Supergrupo Espinhaço é uma espessa seqüência de rochas clásticas dominada por quartzitos, com contribuições subordinadas de rochas vulcânicas e carbonatos, metamorfisada na fácies xisto verde. A maioria dos autores considera estas rochas como a evolução de uma bacia ensiálica (rifte-sinéclise), nucleada por volta de 1,75 Ga (e.g., Babinski et al., 1994; Dussin \& Dussin, 1995; Uhlein et al., 1998; Martins-Neto et al., 2001).

O Supergrupo São Francisco apresenta duas unidades neoproterozóicas: Grupo Macaúbas e Grupo Bambuí. O Grupo Macaúbas é constituído por sedimentos glacio-continentais proximais e distais (aluviais e lacustres) (Pflug \& Renger 1973; Karfunkel \& Hope 1988; Uhlein 1991, Dominguez 1993; Pedrosa Soares et al. 1998; Uhlein et al. 1999; Martins Neto et al. 2001). O Grupo Macaúbas registra o desenvolvimento de

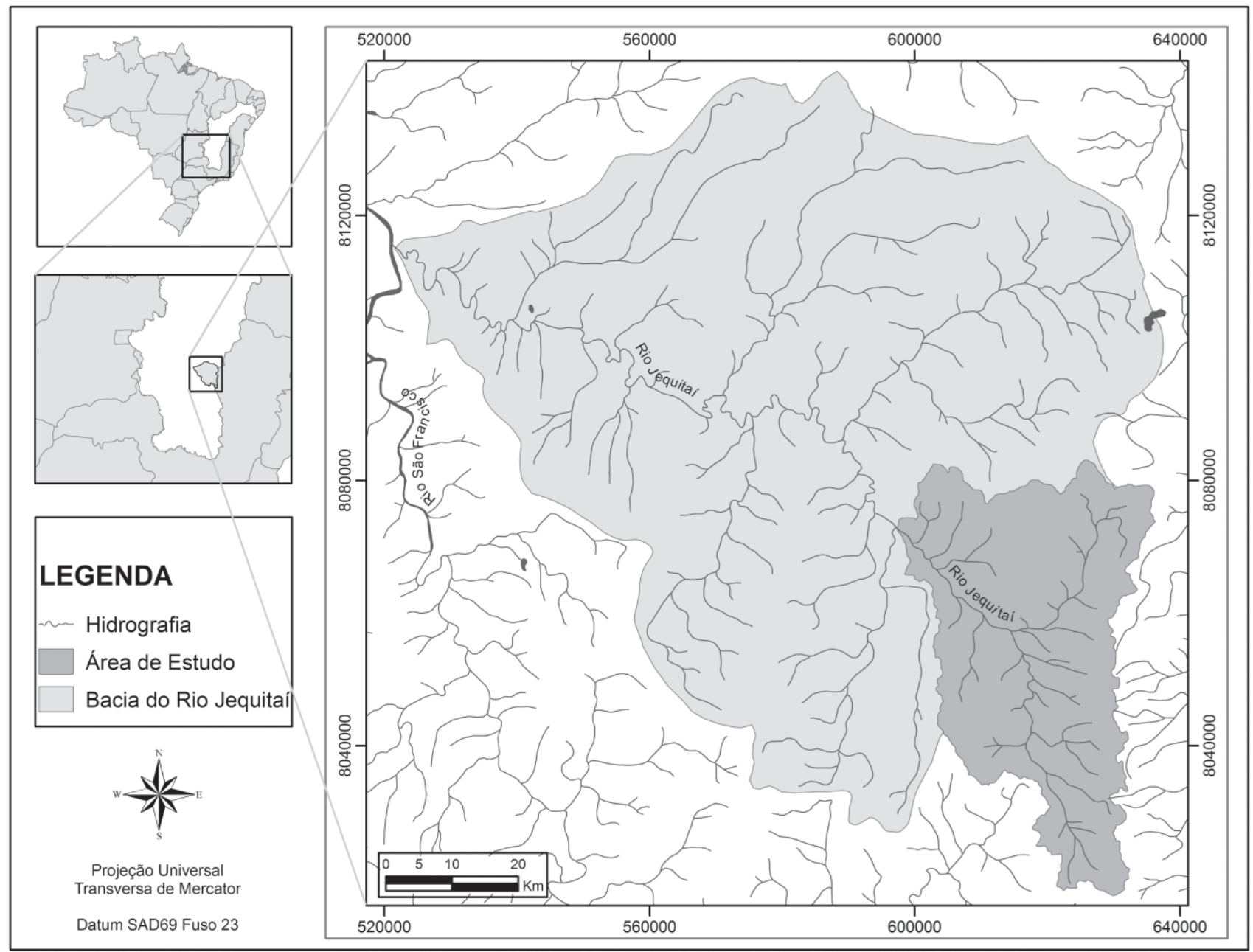

Figura 1. Localização da área de estudo. 


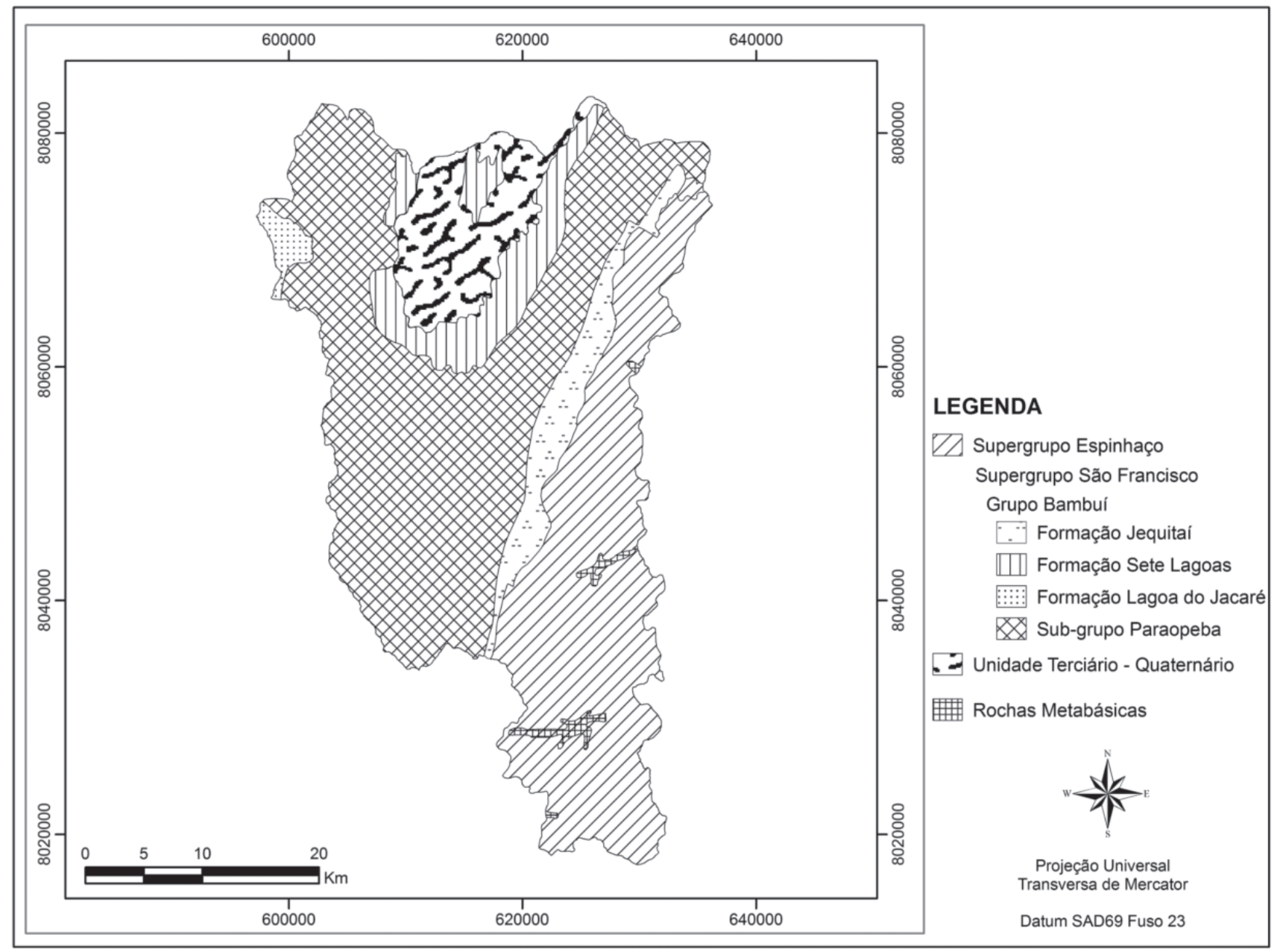

Figura 2. Mapa Geológico (Projeto RADAMBRASIL, 1978).

uma bacia neoproterozóica que evoluiu de um rifte continental (1000 e $880 \mathrm{Ma}$ ) para margem passiva (800 Ma) (Noce et al. 1997; Pedrosa-Soares et al. 1998, 2001; Uhlein et al. 1999; Martins-Neto et al. 2001; Tack et al. 2001; Lima et al. 2002).

O Grupo Bambuí é constituído por depósitos carbonáticos e siliciclásticos. O preenchimento da Bacia do Bambuí é caracterizado por três seqüências transgressivoregressivo cada qual apresentando pelitos e calcilutitos marinho-profundo na base, sobre os quais progridem depósitos marinhos rasos influenciados por tempestades, portadores de estromatólitos (Dardenne, 1981, 2000). Dardenne (1978) estabeleceu uma subdivisão estratigráfica de forma a buscar uma uniformização a partir de correlações litoestratigráficas na bacia, sendo constituída pelas seguintes formações da base para o topo: Jequitaí (ruditos), Sete Lagoas (margas, calcilutitos, calcarenitos, biolititos), Serra de Santa Helena (pelitos), Lagoa do Jacaré (calcarenitos, pelitos), Serra da Saudade (pelitos) e Três Marias (pelitos, arenitos). Na área de estudo estão presentes as formações Sete Lagoas e Lagoa do Jacaré. Com exceção da formação Três Marias, que representa o assoreamento final do foreland Bambuí, todas as demais formações deste Grupo formam o Subgrupo Paraopeba. Sobre estas rochas ocorrem à cobertura do Terciário-Quartenário formado por sedimentos fluviais silto arenoso e areno-siltosos, cobertura argilo-siltosa e siltoarenosa.

As unidades geomorfológicas descritas para a área são: Serra do Espinhaço, Planalto do Jequitinhonha e Depressão São Franciscana (CODEVASF, 1993). A Serra do Espinhaço apresenta predominância de cristas, picos e vales encaixados, e em menor proporção as formas levemente onduladas. $\mathrm{O}$ Planalto do Jequitinhonha apresentam principalmente formas tabulares levemente onduladas, mas são encontradas interflúvios tabulares com vertentes ravinadas e vales 
encaixados. A Depressão São Franciscana tem grande parte de sua área caracterizada por rampas, colinas e morrotes.

\section{Metodologia}

A metodologia empregada para este trabalho pode ser subdividida nas seguintes etapas: (a) construção do MDT hidrologicamente corrigido; (b) delimitação das bacias de drenagem; (c) cálculo dos atributos morfométricos; e (d) análise estatística multivariada.

\subsection{Construção do MDT Hidrologicamente Corrigido}

Na elaboração do MDT da área de estudo foram utilizadas quatro cartas topográficas digitais na escala de 1:100.000 cedidas pela Companhia de Desenvolvimento dos Vales do São Francisco e Parnaíba (CODEVASF). Antes de proceder à interpolação realizou-se uma verificação da existência de erros provenientes do processo de digitalização das cartas topográficas.

A interpolação dos dados para a geração do MDT foi realizado pelo método TOPOGRID desenvolvido para estudos hidrológicos (Hutchinson, 1989). Este método permite congregar tanto as feições hidrográficas como a remoção de dados espúrios relativos a pontos de depressões (Jenson e
Dominique, 1988). Para o presente trabalho o MDT foi gerado com a resolução espacial de 20 metros, referente a acuidade visual de $0,2 \mathrm{~mm}$.

No entanto, ainda observa-se a existência de problemas em áreas muito planas devido à falta de uma direção preferencial do fluxo, dificultando a delimitação automática da rede de drenagem e, consequientemente, das sub-bacias hidrográficas (Garbrecht e Martz, 1997; Tribe, 1992). Para que a delimitação da rede de drenagem ocorra de modo satisfatório foi induzida a direção de fluxo ao longo dos canais a partir do aprofundamento da hidrografia no MDT.

O procedimento para o aprofundamento da drenagem utiliza operações algébricas simples entre o MDT e uma imagem binária da rede hidrográfica onde a largura dos canais é restrita a um pixel. Inicialmente, são gerados dois MDTs: (a) MDT do canal de drenagem com valores altimétricos subtraídos de 50 metros e com as demais áreas com valores nulos; e (b) MDT com valores nulos para o canal de drenagem. A partir da soma destas duas grades é gerado um MDT com aprofundamento de drenagem de 50 metros, valores extremamente baixos em relação às áreas circunvizinhas, o que gera artificialmente uma feição de canyon (Figura 3).

Para determinar a direção do escoamento dos canais ao longo das drenagens foi empregado o método D8 para manter a relação unimodal, ou seja, garantindo que todo o fluxo fosse
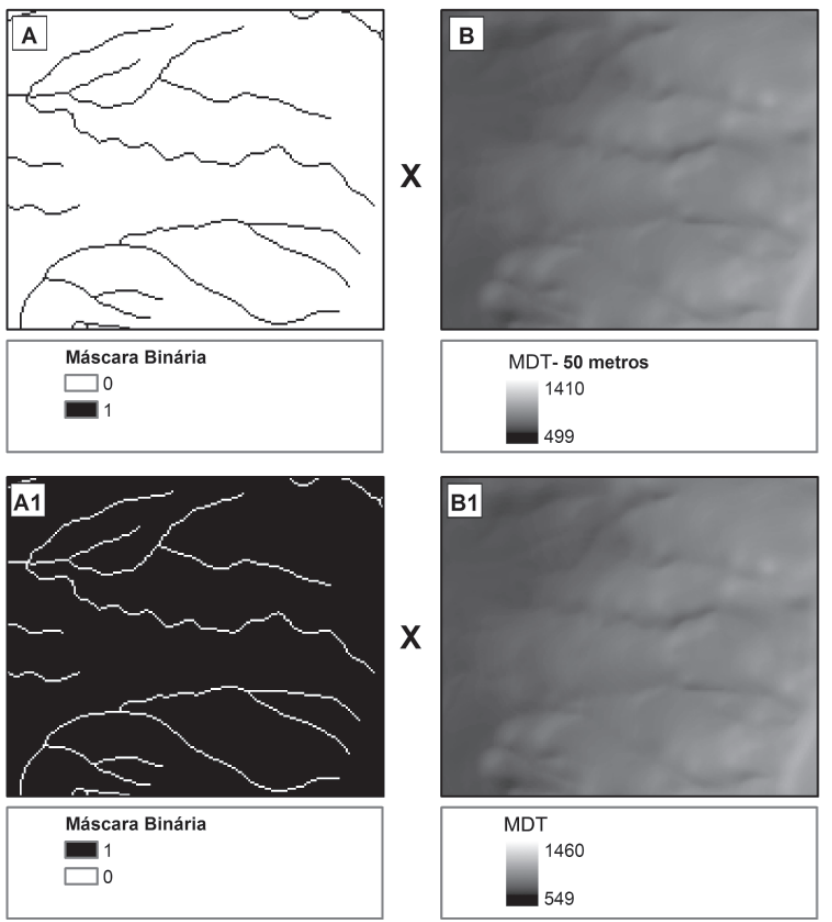
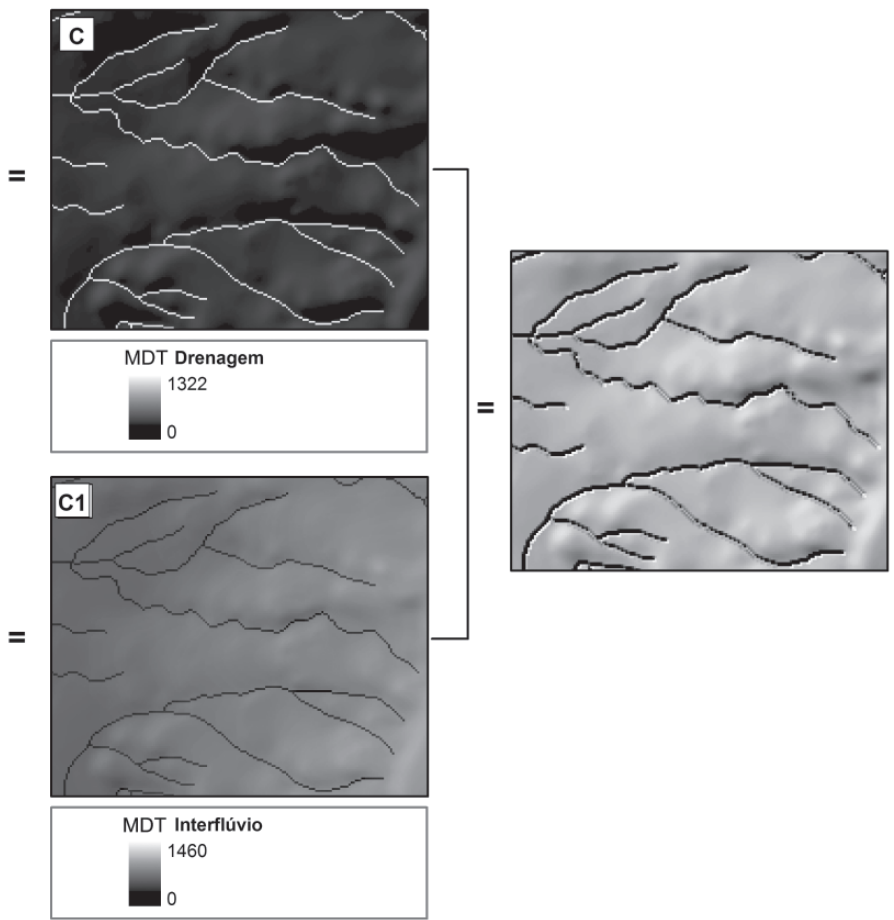

Figura 3. Operações realizadas para o aprofundamento da rede de drenagem apresentando como resultado o MDT hidrologicamente correto. 


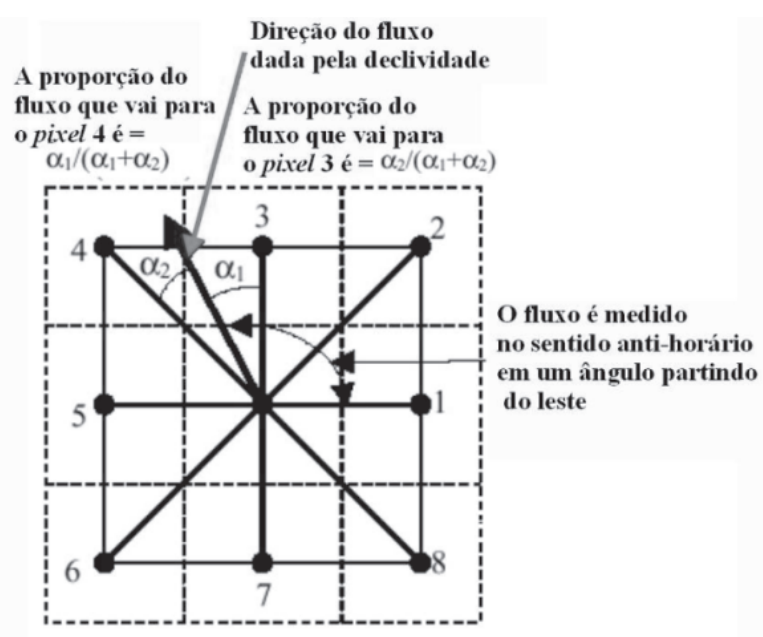

Figura 4. Cálculo da direção de fluxo pelo método D-Infinito adaptado de Tarboton (1997).

transmitido para uma única célula dos seus oito circunvizinhos. Para as demais áreas, a direção de fluxo foi calculada pelo método D-infinito desenvolvido por Tarboton (1997), que também leva em consideração as oito células vizinhas para determinar a orientação do escoamento em cada célula, porém distribui o fluxo proporcionalmente de acordo com a angulação formada pelas facetas triangulares adjacentes a cada célula da matriz (Figura 4).

\subsection{Delimitação das Bacias de Drenagem}

A bacia hidrográfica pode ser subdividida a partir de suas características espacialmente distribuídas. Devido a sua simplicidade e funcionalidade, um dos métodos mais utilizados baseia-se nos critérios de hierarquia fluvial de Strahler (1952). Neste os canais provenientes de nascentes são definidos como de primeira ordem, dando início a uma lógica onde os canais de segunda ordem são formados pela junção de dois canais de primeira ordem; os de terceira ordem são formados pela junção de dois de segunda ordem e assim sucessivamente. Desta forma a ordem da bacia é definida pela mais alta ordem de canal presente.

A rotina TAUDEM (Terrain Analysis Using Digital Elevation Models) desenvolvida por Tarboton (2005) permite obter de forma automatizada as bacias segundo a hierarquização de Strahler. Esta rotina permite individualizar as bacias para cada canal da rede de drenagem utilizando como parâmetro o MDT e os demais atributos gerados, a direção de fluxo, área de contribuição e declividade. $\mathrm{Na}$ existência de áreas não classificadas são gerados fragmentos (Figura 5), demarcados pelo enxutório de duas bacias de mesma ordem.

\subsection{Cálculo dos Atributos Morfométricos das Bacias de Drenagem}

Os dados morfométricos de uma bacia de drenagem podem trazer uma perspectiva dos diversos processos que atuam sobre o modelado. Vários trabalhos estimam de forma indireta as propriedades ambientais de solo, rocha e recursos hídricos a partir da correlação com variáveis morfométricas de bacias hidrográficas, devido à maior facilidade de serem estimadas (Villela \& Mattos, 1975, Cardoso et al. 2006). Além de estimar os fatores físicos esses atributos permitem também entender o processo de ocupação antrópica (Mesa, 2006; Souza \& Batista, 2007).

Neste trabalho, foram considerados os seguintes atributos morfométricos de cada sub-bacia: altimetria, declividade, curvatura, área e perímetro. Além disso, foram calculados os índices: (a) amplitude topográfica, muito utilizado para estudos geomorfológicos (Hadley \& Schumm, 1961; Meis et al., 1982; Silva, 2002, 2005), e (b) índice de circularidade, que apresenta relação com o comportamento da vazão no exutório da bacia (Miller, 1953; Strahler, 1964). Esses atributos foram obtidos a partir do módulo Spatial Analyst no programa ArcGIS 9.2 (ESRI, 2006).

\subsection{Análise Estatística Multivariada das Bacias de Drenagem}

As análises multivariadas favorecem o agrupamento dos dados, permitindo simplificar e organizar o montante de informações levantadas. Dentre os métodos multivariados aplicados no estudo de morfometria de bacias de drenagem destacam-se a análise de grupos e a análise de componentes principais (ACP), ambos os métodos aplicados neste trabalho (Mather \& Doornkamp, 1970; Oliveira, 2007).

Os métodos de análise de grupos empregam classificadores que utilizam, além da informação de cada pixel, a informação espacial que envolve a relação entre os pixels vizinhos, agrupando os dados em classes distintas definidas por intermédio de uma medida de similaridade ou dissimilaridade. Dentre os métodos de análise de grupos, um dos mais utilizados é o K-means onde os elementos são agrupados a partir de centros previamente determinados.

A ACP pode ser sumarizada em três passos (Richards, 1986): (a) derivação da matriz de correlação ou de variânciacovariância, (b) cálculo dos autovetores e autovalores, e (c) transformação linear do conjunto de dados. Esta transformação tem como base a rotação do espaço de atributos na direção dos autovetores com o intuito de tornar ortogonal o conjunto de dados, promovendo a união das informações de maior correlação nas primeiras componentes (Mather, 1999). A APC reduz a dimensionalidade dos dados, concentrando as informações significativas nas primeiras componentes geradas pela transformação (Schowengerdt, 1997). 


\section{Resultados}

\subsection{Resultados das Bacias de Drenagem e dos Dados de Morfometria}

Foram delimitados automaticamente 1.773 bacias mais fragmentos de $1^{\mathrm{a}}$ ordem fluvial, 536 de $2^{\mathrm{a}}$ ordem fluvial e 136

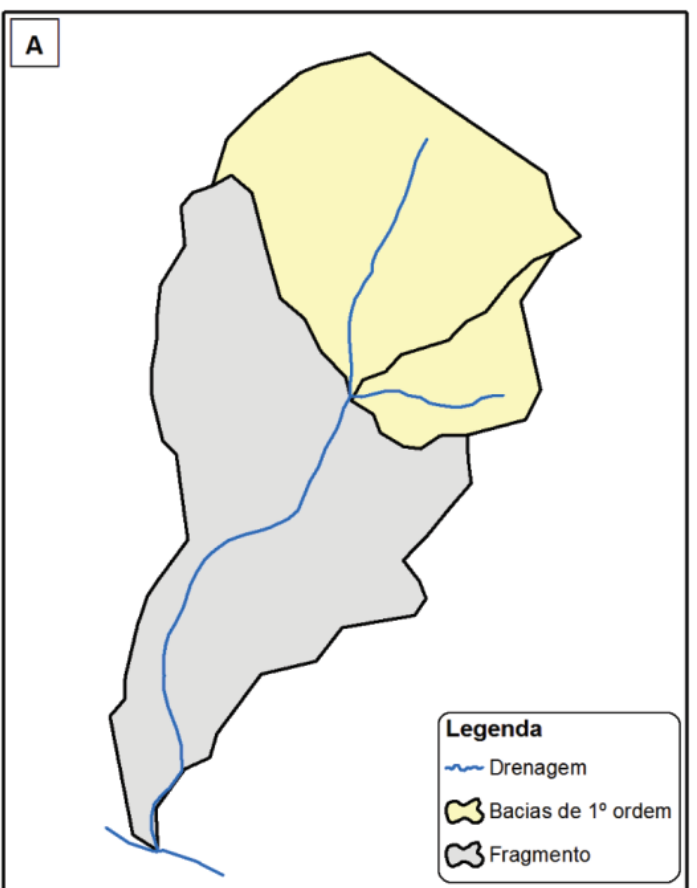

de $3^{\text {a }}$ ordem fluvial (Figura 6). O estudo das diferentes ordens fluviais torna-se importante para a compreensão do efeito da variação da escala na aglutinação das informações.

A distribuição espacial dos atributos morfométricos demonstra visualmente uma subdivisão de setores na bacia hidrográfica com segmentação de grupos de leste para oeste

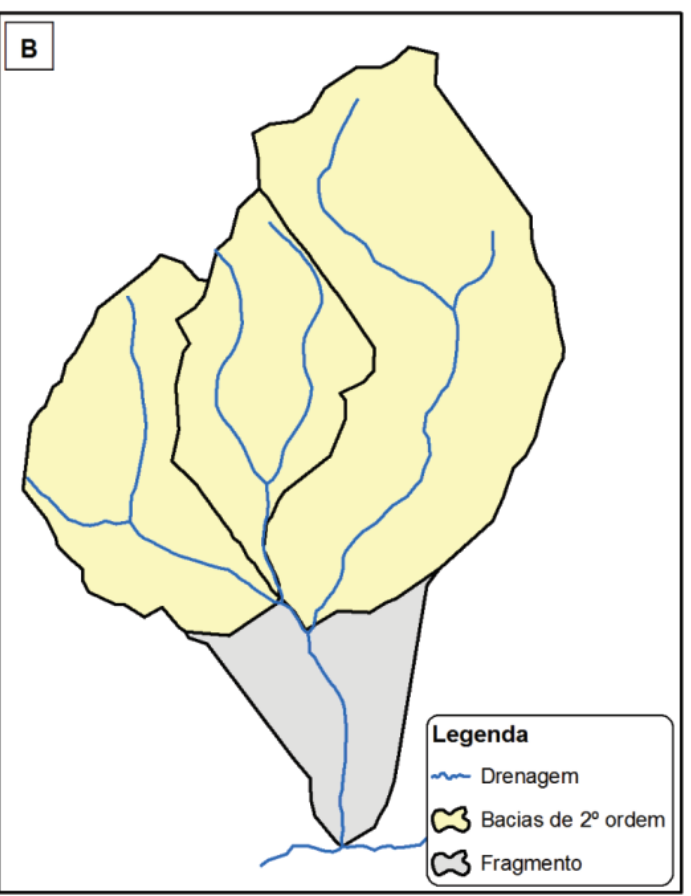

Figura 5. Bacias de $1^{a}$ ordem e seus fragmentos da hierarquia de Strahler (A) e bacias de $2^{a}$ ordem e seus fragmentos da hierarquia de Strahler $(B)$.
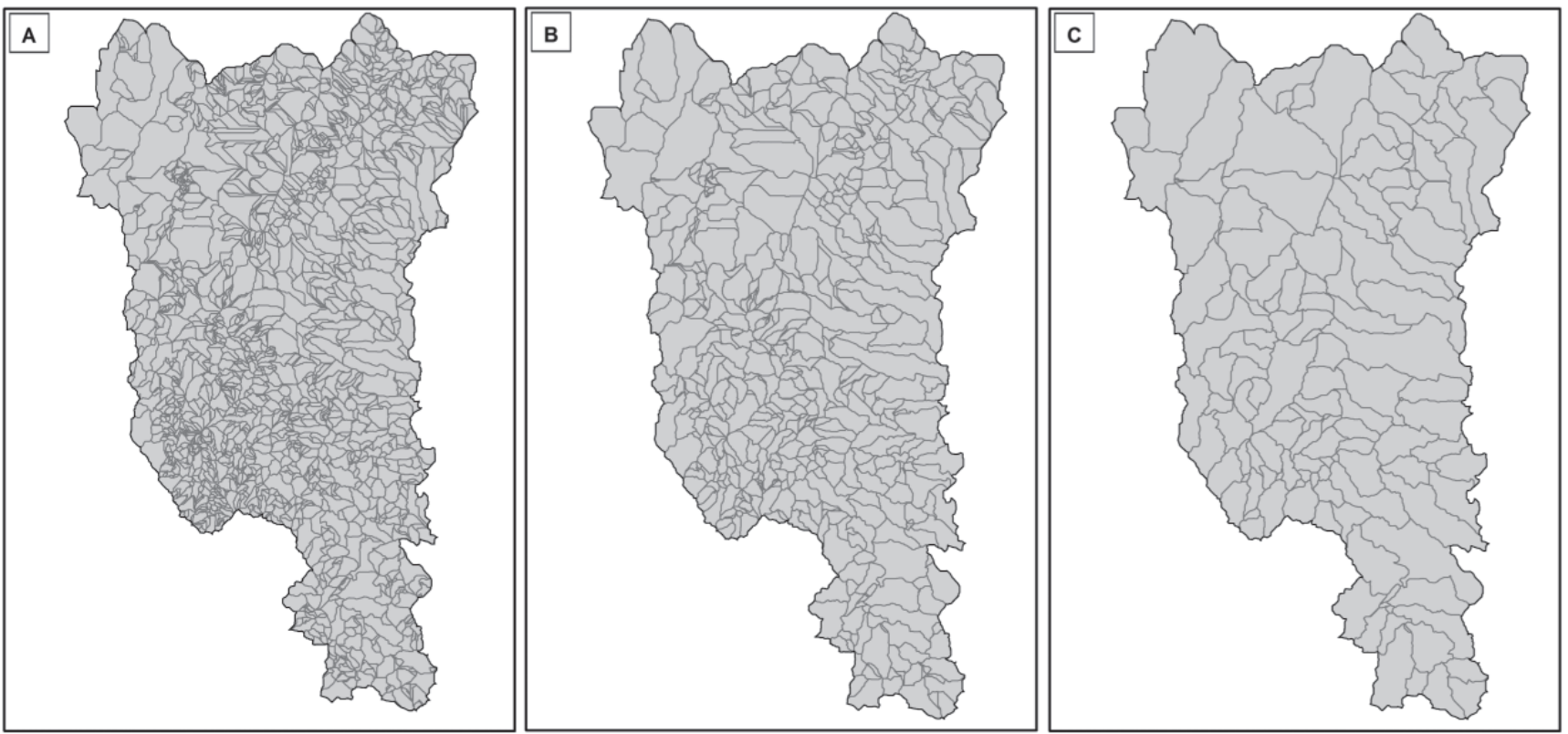

Figura 6. Bacias de drenagem e fragmentos de $1^{a}$ ordem (A), $2^{a}$ ordem $(B)$ e $3^{a}$ ordem $(C)$ para a área do Alto Jequitaí. 


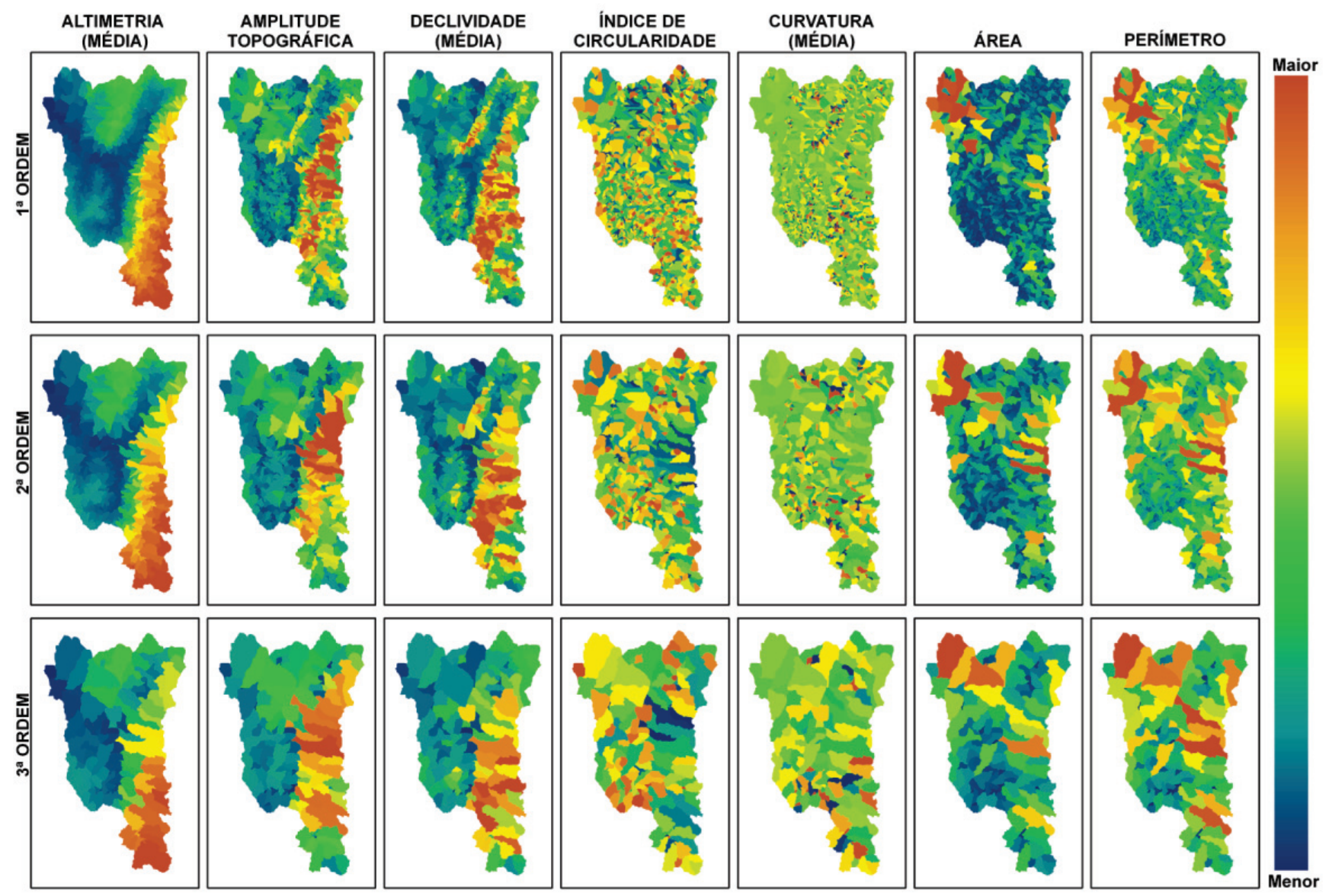

Figura 7. Atributos morfométricos das bacias de drenagem de $1^{a}, 2^{a}$ e $3^{a}$ ordem fluvial (altimetria média, amplitude topográfica, declividade média, índice de circularidade, curvatura, área e perímetro).

(Figura 7). Estes atributos apresentam uma distribuição semelhante, destacando os três principais compartimentos geomorfológicos descritos para a área em estudo. Esta diferenciação é mais visível nas bacias de menor ordem fluvial.

Os atributos de altimetria, amplitude topográfica e declividade apresentam de modo geral um crescimento da correlação com o aumento da ordem fluvial (Tabelas 1, 2 e 3). O setor dominado pela Serra do Espinhaço, na porção leste da bacia, apresenta um comportamento diferenciado com a predominância de valores mais altos de altimetria, amplitude topográfica e declividade. Em contraposição, a parte oeste da bacia apresenta áreas mais baixas e planas, com menor amplitude topográfica e declividade, caracterizando a Depressão São Franciscana. Na parte central ao norte da bacia esses parâmetros morfométricos evidenciam a unidade do Planalto do Jequitinhonha com formas tabulares levemente onduladas, caracterizada por uma faixa altimétrica intermediária com baixos valores de declividades e amplitude topográfica no topo que progressivamente aumenta em direção a sua borda oriental.
O parâmetro de curvatura apresenta uma baixa correlação com os demais atributos e evidencia as feições de bordas. Essas feições se caracterizam pelo aumento da curvatura e desnível abrupto descrito nas bacias ao longo das áreas de dissecação do Planalto do Jequitinhonha e da Serra do Espinhaço. Nas bacias de $1^{\text {a }}$ ordem a curvatura evidencia as áreas de borda, formando uma faixa delgada que delimita os limites dos domínios geomorfológicos.

Os parâmetros área e perímetro apresentam-se a mais alta correlação entre os atributos analisados. Estes parâmetros evidenciam um comportamento diferenciado ao noroeste da bacia, onde se desenvolvem as maiores bacias hidrográficas com presença de planícies aluvionares e terraços fluviais.

O índice de circularidade conjuntamente com a curvatura são os atributos menos correlacionáveis. No entanto é possível verificar um padrão de distribuição desse atributo. As áreas da Serra do Espinhaço observam-se bacias mais alongadas com baixos índices de circularidade. Na parte centro-sul se observa a presença de bacias com maiores índices de circularidade, localizadas nas porções mais deprimidas do relevo. 
Tabela 1 - Matriz de correlação dos parâmetros morfométricos para bacias de $1^{a}$ ordem e fragmentos.

\begin{tabular}{|c|c|c|c|c|c|c|c|}
\hline $\begin{array}{c}\text { Atributos } \\
\text { Morfométricos de } \\
\text { Bacias } \\
\text { Hidrográficas }\end{array}$ & 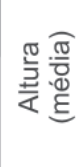 & 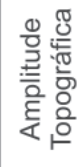 & 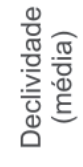 & 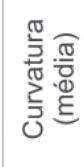 & 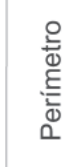 & $\stackrel{\mathbb{d}}{\frac{\mathbb{d}}{4}}$ & 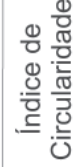 \\
\hline & 1,00 & 0,40 & 0,41 & 0,05 & 0,12 & 0,08 & 0,06 \\
\hline & 0,40 & 1,00 & 0,68 & 0,04 & 0,51 & 0,37 & $-0,02$ \\
\hline $\begin{array}{l}\text { Declividade } \\
\text { (média) }\end{array}$ & 41 & 0,68 & 1,00 & $-0,40$ & $-0,03$ & $-0,02$ & 0,17 \\
\hline $\begin{array}{l}\text { Curva } \\
\text { (méc }\end{array}$ & 05 & 04 & $-0,04$ & 1,00 & 0,07 & 0,04 & 0,04 \\
\hline Períme & 12 & 0,51 & $-0,03$ & $-0,03$ & 1,00 & 0,88 & $-0,16$ \\
\hline Área & 08 & 0,37 & $-0,02$ & $-0,02$ & 0,88 & 1,00 & 0,04 \\
\hline $\begin{array}{l}\text { Índice de } \\
\text { Circularidade }\end{array}$ & 0,06 & $-0,02$ & 0,17 & 0,17 & $-0,16$ & 0,04 & 1,00 \\
\hline
\end{tabular}

Tabela 2 - Matriz de correlação dos parâmetros morfométricos para bacias de $2^{a}$ ordem e fragmentos.

\begin{tabular}{|c|c|c|c|c|c|c|c|}
\hline $\begin{array}{c}\text { Atributos } \\
\text { Morfométricos de } \\
\text { Bacias } \\
\text { Hidrográficas }\end{array}$ & 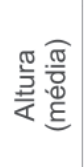 & 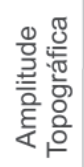 & 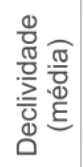 & 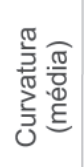 & 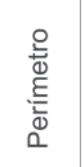 & 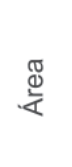 & 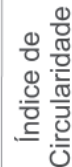 \\
\hline & 1,00 & 0,49 & 0,53 & 0,07 & 0,22 & 0,19 & 0,07 \\
\hline & 0,49 & 1,00 & 0,73 & 0,03 & 0,59 & 0,49 & $-0,04$ \\
\hline $\begin{array}{l}\text { Declividade } \\
\text { (média) }\end{array}$ & 0,53 & 0,73 & 1,00 & $-0,01$ & 0,12 & 0,08 & 0,14 \\
\hline $\begin{array}{l}\text { Curvatura } \\
\text { (média) }\end{array}$ & 07 & ,03 & $-0,01$ & 1,00 & 0,06 & 0,05 & $-0,03$ \\
\hline Períme & ,22 & 0,59 & 0,12 & 0,06 & 1,00 & 0,92 & $-0,11$ \\
\hline Área & 0,19 & 0,49 & 0,08 & 0,05 & 0,92 & 1,00 & 0,06 \\
\hline $\begin{array}{l}\text { Índice de } \\
\text { Circularidade }\end{array}$ & 0,07 & $-0,04$ & 0,14 & $-0,03$ & $-0,11$ & 0,06 & 1,00 \\
\hline
\end{tabular}

\section{2 - Resultados da Análise de Componentes Principais (ACP)}

A análise multivariada de ACP permite a diminuição da redundância de dados concentrando as informações em poucas componentes. Os dados morfométricos das bacias de $1^{\mathrm{a}}, 2^{\mathrm{a}}$ e $3^{\mathrm{a}}$ ordem fluvial foram processados separadamente. A transformação ACP utilizou a matriz de correlação de forma a normalizar as diferenças existentes entre os valores dos atributos morfométricos. Os autovalores relativos às três primeiras componentes principais, nas diversas ordens
Tabela 3 - Matriz de correlação dos parâmetros morfométricos para bacias de $3^{a}$ ordem e fragmentos.

\begin{tabular}{|c|c|c|c|c|c|c|c|}
\hline $\begin{array}{c}\text { Atributos } \\
\text { Morfométricos de } \\
\text { Bacias } \\
\text { Hidrográficas }\end{array}$ & 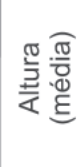 & 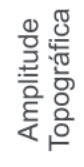 & 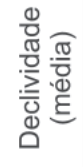 & 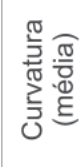 & 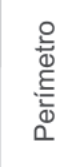 & 选 & 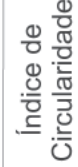 \\
\hline & 1,00 & 0,52 & 0,62 & 0,00 & 0,28 & 0,20 & $-0,19$ \\
\hline & 0,52 & 1,00 & 0,70 & $-0,08$ & 0,67 & 0,52 & $-0,37$ \\
\hline $\begin{array}{c}\text { Declividad } \\
\text { (média) }\end{array}$ & 62 & 0,70 & 1,00 & 0,11 & 0,23 & 0,14 & 0,12 \\
\hline $\begin{array}{l}\text { Curvatura } \\
\text { (média) }\end{array}$ & 00 & $-0,08$ & 0,11 & 1,00 & $-0,14$ & $-0,09$ & $-0,12$ \\
\hline Perímetro & 0,28 & 0,67 & 0,23 & $-0,14$ & 1,00 & 0,93 & $-0,4$ \\
\hline Área & 0,20 & 0,52 & 0,14 & $-0,09$ & 0,93 & 1,00 & $-0,2$ \\
\hline $\begin{array}{l}\text { Índic } \\
\text { Circula }\end{array}$ & $-0,19$ & $-0,37$ & $-0,12$ & $-0,12$ & $-0,41$ & $-0,23$ & 1,0 \\
\hline
\end{tabular}

fluviais, atingem praticamente valores de $90 \%$ da variância total, o que demonstra uma alta concentração de informação nestas componentes.

A primeira componente principal $(\mathrm{CP})$ nas diferentes ordens fluviais foi influenciada principalmente pelos atributos de altimetria (Figura 8). Torna-se evidente a presença das três unidades geomorfológicas para as diferentes ordens fluviais. A Serra do Espinhaço apresentam os mais altos valores, enquanto a Depressão São Franciscana os mais baixos e o Planalto do Jequitinhonha os valores intermediários.

A segunda $\mathrm{CP}$ apresenta maior influência dos atributos de amplitude topográfica, declividade média e curvatura. Nesta componente, as bacias de primeira ordem fluvial destacam as áreas de bordas tanto da Serra do Espinhaço como do Planalto do Jequitinhonha. As bacias referentes à segunda e terceira ordem fluvial não apresentam de forma tão evidente as bordas mas permitem evidenciar padrões referentes as áreas de maior declividade relativos a Serra do Espinhaço.

A terceira CP demonstra maior influência da área, perímetro e circularidade. Observa-se que os maiores valores destas componentes são referentes às bacias de maior perímetro e área enquanto que os menores valores são das bacias com menor dimensão e índices de circularidades.

\section{3 - Resultados da Análise de Grupos}

No presente trabalho a análise de grupos foi empregada utilizando o método K-Means, para todo o conjunto de variáveis morfométricas, considerando o número máximo de sete classes nas diferentes ordens fluviais (Figura 9). 
Sodré, R.V.R. et al.

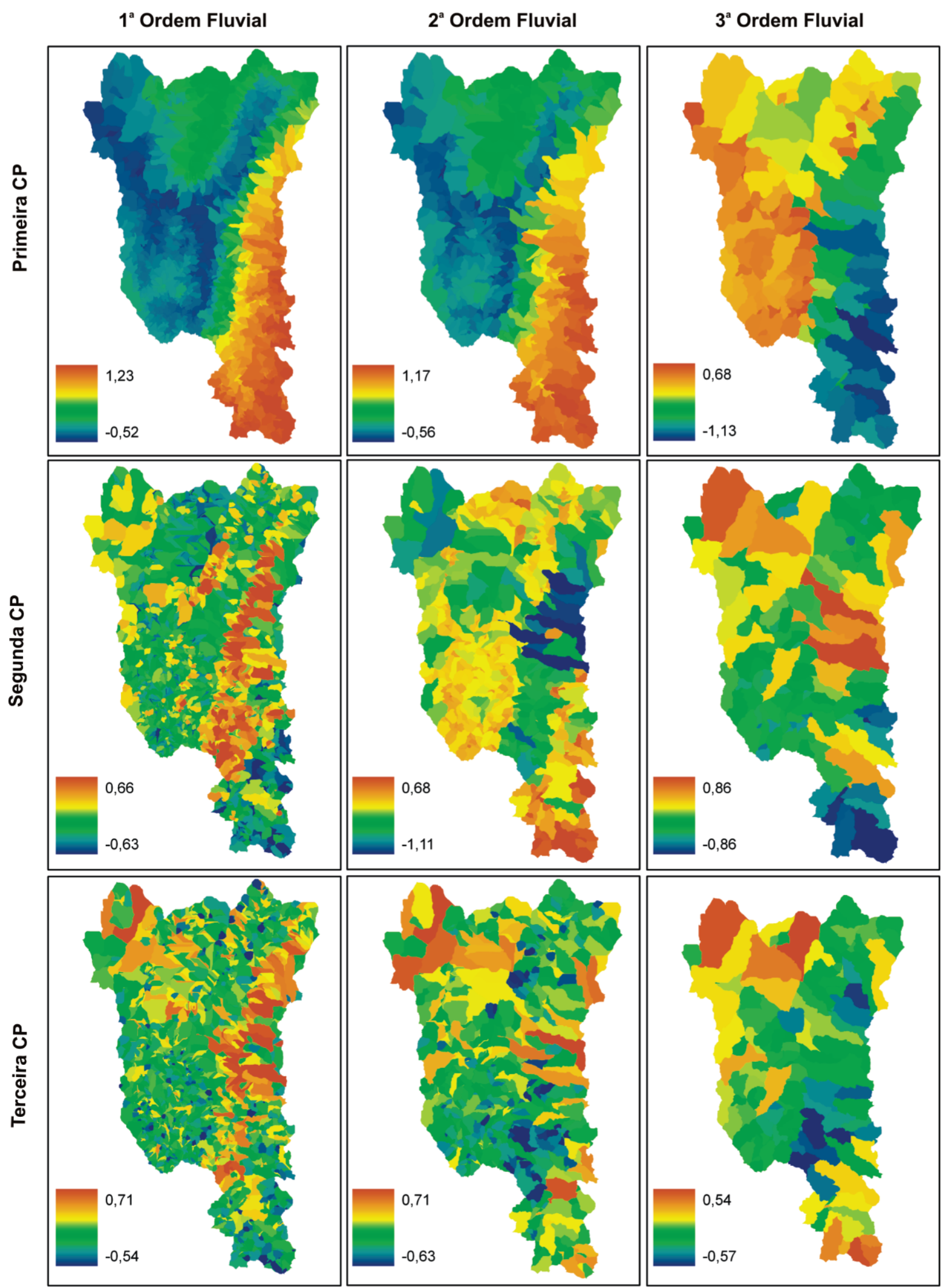

Figura 8. As três primeiras Componentes Principais referentes às bacias de drenagem relativas à $1^{a}, 2^{a}$ e $3^{a}$ ordem fluvial. 

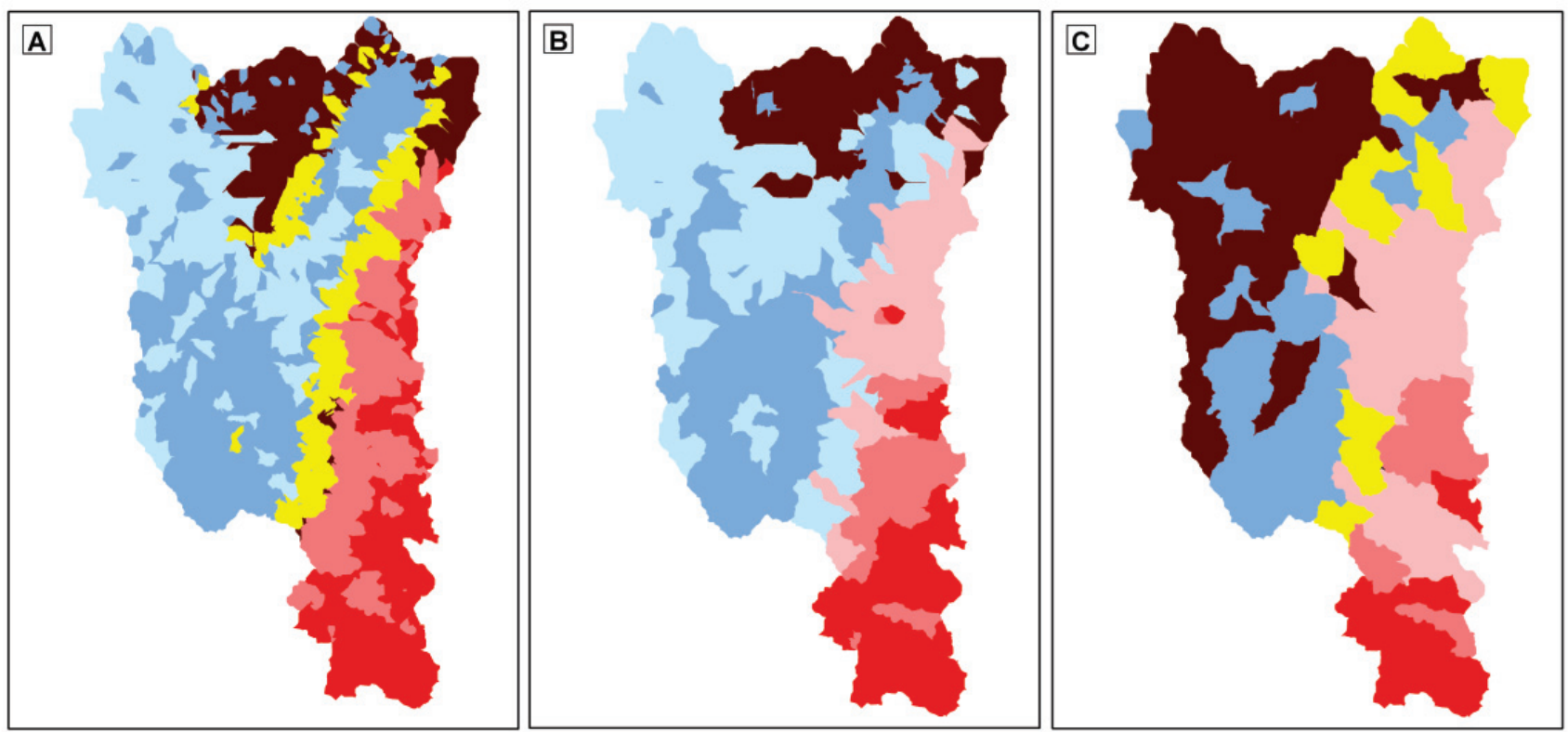

Legenda
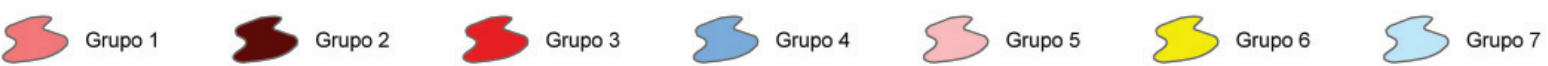

Figura 9 - Classificação pelo Método K-Means das bacias de drenagens de $1^{a}$ ordem fluvial (A), $2^{a}$ ordem fluvial (B) e $3^{a}$ ordem fluvial $(C)$.

As classes obtidas para as bacias de primeira ordem fluvial destacam as principais unidades geomorfológicas: Serra do Espinhaço (grupo 1 e 3), Planalto do Jequitinhonha (grupo 2), Depressão São Franciscana(grupo 4 e 7) e uma classe referente às áreas de bordas caracterizadas por altas declividades provenientes da dissecação do planalto e da serra (grupo 6). Dentro das unidades geomorfológicas principais pode-se verificar uma subdivisão interna que demonstram variações topográficas e de dimensões de bacias.

$\mathrm{Na}$ classificação das bacias de segunda ordem preservam-se ainda as feições provenientes das três unidades geomorfológicas, mas ocorre a supressão da classe de borda. Essa característica é devido à maior dimensão das bacias que não ficam contidas especificamente nesta porção do relevo.

O efeito da diminuição da escala, com o aumento da dimensão da bacia, torna-se mais evidente para as bacias de terceira ordem, onde se observa uma mescla das unidades do Planalto do Jequitinhonha e a Depressão do São Francisco. Nesta ordem fluvial das bacias de drenagem os limites geomorfológicos não ficam bem delimitados.

\section{Conclusões}

O conhecimento geomorfológico é de fundamental importância no diagnóstico das condições ambientais, contribuindo para nortear as atividades humanas. Assim, as bacias de drenagem estabelecem unidades de estudo apropriadas para estabelecer técnicas de manejo e conservação que visam promover a proteção de água, solo e outros recursos naturais, essenciais à sustentabilidade da atividade econômica, controle da degradação ambiental e à eqüidade social (Lanna, 1995).

A metodologia adotada no presente trabalho possibilitou segmentar as bacias de drenagem a partir do grau de similaridade existente entre os seus atributos morfométricos. Neste propósito os métodos estatísticos multivariados (APC e análise de grupos) mostraram-se adequados evidenciando padrões espaciais que refletem dinâmicas ambientais similares. A abordagem considerando as três diferentes ordens fluviais de Strahler permitiu analisar o fator escalar.

Os dados morfométricos permitiram distinguir comportamentos distintos entre as bacias, que enfatizaram as unidades geomorfológicas: Serra do Espinhaço, Depressão São Franciscana e Planalto do Jequitinhonha. As diferenças encontradas pela análise morfométrica reflete comportamentos distintos de material de origem e dinâmica da água superficial peculiar de cada unidade.

A Depressão São Franciscana é caracterizada por áreas baixas e planas, provenientes em sua maior extensão de rochas do Grupo Paraopeba, que se apresentam cobertas por sedimentos fluviais silto-arenosos e areno-siltosos como também, cobertura argilo-siltosa e silto-arenosa. Na Depressão São Franciscana observam-se planícies aluvionares, terraços 
fluviais, rampas onduladas e colinas. Dentro dessa unidade é possível distinguir diferentes padrões provenientes de pequenas variações, como o setor noroeste caracterizado por ser mais plano e conter bacias com maiores dimensões em área e perímetro.

O Planalto do Jequitinhonha mostra-se com altimetria intermediária com áreas planas no topo e bordas escarpadas contendo alta declividade e curvatura. Esta unidade está localizada sobre rochas da Formação Sete Lagoas do Grupo Bambuí.

A unidade geomorfológica da Serra do Espinhaço localiza-se sobre o Supergrupo geológico homônimo. Esta unidade é constituída principalmente por rochas clásticas dominada por quartzitos, resistentes ao processo erosivo, que configuram um relevo com altas altitudes, declividades e amplitude topográfica.

A metodologia adotada utilizando o sistema de informações geográficas mostrou-se adequada para o tratamento do conjunto de informações morfométricas das bacias evidenciando padrões similares.

\section{Referências Bibliográficas}

Babinski, M.; Brito-Neves, B.B.; Machado, N.; Noce, C.M.; Uhlein, A. \& Van Schmus, W.R. (1994) Problemas da metodologia U-Pb em rochas vulcânicas continentais: Caso do Grupo Rio dos Remédios, Supergrupo Espinhaço, no Estado da Bahia. In: SBG, Congresso Brasileiro de Geologia, 38., Camboriú. Resumos Expandidos. SBG. 2: 409-410.

Cardoso, C.A.; Dias, H.C.T.; Soares, C.P.B. \& Martins, S.V. (2006) Caracterização morfométrica da bacia hidrográfica do rio Debossan, Nova Friburgo, RJ. Revista Árvore, 30 (2): 241-248.

Casseti, V. (1981) Estrutura e gênese da compartimentação da paisagem de Serra Negra (MG). Goiânia: Editora da UFG, $124 \mathrm{p}$.

Christofoletti, A. (1969) Análise morfométrica de bacias hidrográficas. Notícia Geomorfológica, n. 18, p. 3564.

Christofoletti, A. (1970) Análise morfométrica de bacias hidrográficas no Planalto de Poços de Caldas. 375 f. Tese (Livre Docência) - Universidade Estadual Paulista, Rio Claro.

Christofoletti, A. (1977) A mecânica do transporte fluvial. Geomorfologia, 51: 1-42.

Christofoletti, A. (1978) Morfologia de bacias de drenagem. Notícia Geomorfológica, 18: 130-132.
Christofoletti, A. (1980) Geomorfologia. 2. ed. São Paulo: Edgard Blucher. $188 \mathrm{p}$.

CODEVASF. (1993) Projeto Jequitaí: estudos de impacto ambiental - EIA. Aproveitamentos múltiplos de Jequitaí I e II e perímetro de irrigação. Brasília: CODEVASF, v.1, 512p.

Coelho Netto, A.L. (1995) Hidrologia de encosta na interface com a geomorfologia. In: Guerra, A.J.T.; Cunha, S.B. da (Org). Geomorfologia: uma atualização de bases e conceitos. 2. ed. Rio de Janeiro: Bertrand Brasil, $458 \mathrm{p}$.

Dardenne, M.A. (1978) Síntese sobre a estratigrafia do Grupo Bambuí no Brasil central. In: Congresso Brasileiro de Geologia, 30., Recife. Anais. Recife: SBG. 2: 597610.

Dardenne, M.A. (1981) Os grupos Paranoá e Bambuí na Faixa Dobrada Brasília. In: Simpósio sobre o Craton São Francisco e Suas Faixas Marginais, Salvador - BA. Anais. Salvador: SBG-Nucleo BA. 140-157.

Dardenne, M.A. The Brasilia Fold Belt.. In: Cordani, U.G.; Milani, E.J.; Thomaz Filho, A. \& Campos, D.A.. (Org.). Tectonic Evolution of South America. 1 ed. Rio de Janeiro, 231-263

Dominguez, J.M.L. (1993) As coberturas do Cráton do São Francisco: uma abordagem do ponto de vista da análise de bacias. In: Domingues J.M.L. \& Misi, A. (Eds.). O Cráton do São Francisco. SBG/BA-SE, SGM/BA, 137-159.

Doornkamp, J.C. \& King, C.A.M. (1971) Numerical analysis in geomorphology: An introduction. London: Edward Arnold Ltd., 372p.

Dussin I.A. \& Dussin T.M. (1995) Supergrupo Espinhaço: Modelo de evolução geodinâmica. Geonomos, 3(1):19-26.

ESRI. (2006) ArcGIS 9.2. Environmental Systems Research Institute, Redlands, CA.

Freitas, R.O. (1952) Textura de drenagem e sua aplicação geomorfológica. Boletim Paulista de Geografia, 11:53-57.

Garbrecht, J. \& Martz, L.W. (1997) The assignment of drainage direction over flat surfaces in raster digital elevation models. Journal of Hydrology, 193: 204213.

Giles, P.T. \& Franklin, S.E. (1998) An automated approach to the classification of the slope units using digital data. Geomorphology, 21:251-264. 
Horton, R.E. (1945) Erosinal development of streams and their drainage basins: hidrophysical approach to quantitative morphology. Bulletin of the Geological Society of America, 56: 275-370.

Hutchinson, M.F. (1989) A new procedure for gridding elevation and stream line data with automatic removal of spurious pits. Journal of Hydrology, 106: 211-232.Jenson e Dominique, 1988

Karfunkel, J. \& Hoppe, A. (1988) Late Proterozoic glaciation in Central-Eastern Brazil: synthesis and model. Palaeogeography, Palaeoclimatology, Palaeoecology, 65: 1-21.

Lanna, A.E.L. (1995) Gerenciamento de Bacia Hidrográfica. Aspectos Conceituais e Metodológicos. Brasília: IBAMA. $170 \mathrm{p}$.

Lima, S.A.A.; Martins-Neto, M.A.; Pedrosa-Soares, A.C.; Cordani, U.G. \& Nutman A. (2002) A Formação Salinas na área-tipo, NE de Minas Gerais: Uma proposta de revisão da estratigrafia da Faixa Araçuaí com base em evidências sedimentares, metamórficas e idades U-Pb SHRIMP. Revista Brasileira de Geociências, 32: 491-500.

Lima, W.P. (1986) Princípios de hidrologia florestal para o manejo de bacias hidrográficas. São Paulo: Escola Superior de Agricultura Luiz de Queiroz - ESALQ. 242p.

Martins-Neto, M.A.; Pedrosa-Soares, A.C. \& Lima, S.A.A. (2001) Tectonosedimentary evolution of sedimentary basins from Paleoproterozoic to Late Neoproterozoic in the São Francisco craton and Araçuaí fold belt, eastern Brazil. Sedim. Geol., 141142:343-370.

Mather, P.M. \& Doornkamp, J.C. (1970) Multivariate Analysis in Geography with Particular Reference to Drainage-Basin Morphometry. Transactions of the Institute of British Geographers, 51, 163-187.

Mather, P.M. (1999) Computer processing of remotely sensed images. New York: John Wiley \& Sons,Inc., 292 p.

Meijerink, A.M.J. (1988) Data acquisition and data capture through terrain mapping units. ITC Journal, 1: 2344.

Mesa, L.M. (2006) Morphometric analysis of a subtropical Andean basin (Tucumán, Argentina). Environmental Geology, 50: 1235-1242.

Miliaresis, G. \& Argialas, D. (1999) Segmentation of physiographic features from the global digital elevation model/GTOPO30. Computers and Geosciences, 25 (7): 715-728.
Moore, I. D.; O’loughlin, E. M. \& Burch, G. J. (1988) A counterbased topographic model for hydrological and ecological applications. Earth Surface and Processes and Landforms, 13: 305 - 320.

Noce, C.M.; Silva, L.C.; Pedrosa-Soares, A.C.; Alkmim, F.F. \& Peres, G.G. (2003) O embasamento da Faixa Araçuaí no Bloco Guanhães: Dados geocronológicos e evolução. In: Simpósio de Geologia de Minas Gerais, 12, Ouro Preto. Anais, SBG, p.90.

Oliveira S.N.; Carvalho Júnior, O.A. Silva, T.M., Gomes, R.A.T.; Martins, E.S.; Guimarães, R.F. \& Silva, N.C. (2007) Delimitação automática de bacias de drenagens e análise multivariada de atributos morfométricos usando modelo digital de elevação hidrologicamente corrigido. Revista Brasileira de Geomorfologia, 8 (1): 3-21.

Pedrosa-Soares, A.C.; Noce, C.M.; Wiedemann, C.M. \& Pinto, C.P. (2001) The Araçuaí-West-Congo Orogen in Brazil: An overview of a confined orogen formed during Gondwanaland assembly. Precambrian Research, 110: 307-323.

Pedrosa-Soares, A.C.; Vidal, P.; Leonardos, O.H. \& BritoNeves B.B. (1998) Neoproterozoic oceanic remnants in eastern Brazil: Further evidence and refutation of an exclusively ensialic evolution for the Araçuaí-West Congo Orogen. Geology, 26: 519522.

Pflug, R. \& Renger F. (1973) Estratigrafia e evolução geológica da margem SE do Cráton do São Francisco. In: Congresso Brasileiro de Geologia, 27., Aracaju. Anais. SBG. 2: 5-19

Richards, J.A. (1986) Remote sensing digital images analysis: An introduction. Berlin: Springer-Verlag. 281p.

Schowengerdt, R.A. (1997) Remote Sensing: Models and Methos for Image Processing. 3. ed. London: Academic Press. 560 p.

Schumm S.A. (1956) Evolution of drainage systems and slopes in badlands at Perth Amboy, New Jersey. Geological Society of America Bulletin. 67: 597646.

Selby, M.J. (1985) Earth's Changing Surface: An Introduction to Geomorphology. Oxford: Clarendon Press. 607 p.

Silveira, A.L.L. (2001) Ciclo hidrológico e bacia hidrográfica. In: Tucci, C.E.M. (Org.). Hidrologia: ciência e aplicação. São Paulo: EDUSP, p 35-51.

Souza, A.K.P.S. \& Batista, G.T. (2007) Caracterização fisiográfica da bacia hidrográfica do Alto Rio 
Jamanxim, Pará, Brasil. Revista Ambiente e Água, 2(2): 69-87.

Strahler, A. N. (1957) Quantitative analysis of watershed geomorphology. Transactions of the American Geophysical Union (38):913-920.

Strahler, A.N. (1952) Hypsometric (area-altitude) analysis and erosional topography. Bulletin of the Geological Society of America, 63 (10):1117-1142.

Tack L., Wingate M.T.D., Liégeois J.P., Fernandez-Alonso M. \& Deblond A. (2001) Early Neoproterozoic magmatism (1000-910 Ma) of the Zadinian and Mayumbian groups (Bas-Congo): Onset of Rodinia rifting at the western edge of the Congo craton. Precambrian Research, 110: 277-306.

Tarboton, D.G. (2005) Terrain Analysis Using Digital Elevation Models (TauDEM). http:// hydrology.neng.usu.edu/taudem/. Acesso em 05/ 02/2008.

Texeira, A. L. A.; Moretti, E. \& Cristofoletti A. (1992) Introdução aos sistemas de informação geográfica. Rio Claro: Edição do Autor, 79 p.

Tolentino, M.; Gandolfi, N. \& Paraguassu, A.B. (1968) Estudo morfométrico das bacias hidrográficas do Planalto de São Carlos - SP. Revista Brasileira de Geografia, 30 (4): 42-50.

Tribe, A. (1992) Automated recognition of valley lines and drainage networks from grid digital elevation models: A review and a new method. Journal of Hydrology, 139: 263-293.

Uhlein A. (1991) Transição cráton-faixa dobrada: Exemplo do Cráton doSão Francisco e da Faixa Araçuaí (Ciclo Brasiliano) no Estado deMinas Gerais: Aspectos estratigráficos e estruturais. $295 \mathrm{f}$. Tese (Doutorado) - Instituto de Geociências, Universidade de São Paulo, São Paulo.

Uhlein, A.; Trompette, R.R. \& Alvarenga, C.J.S. (1999) Neoproterozoic glacial and gravitational sedimentation on a continental rifted margin: The Jequitaí-Macaúbas sequence (Minas Gerais, Brazil). Journal of South American Earth Sciences, 12:435-451.

Uhlein, A.; Trompette, R.R. \& Egydio-Silva, M. (1998) Proterozoic rifting and closure, SE border of the São Francisco Craton, Brazil. Journal of South American Earth Sciences, 11:191-203.

Villela, S.M.; \& Mattos, A. (1975) Hidrologia Aplicada. São Paulo: McGraw-Hill. 245p. 